OPEN ACCESS

Edited by:

Kai Breuhahn,

Universität Heidelberg, Germany

Reviewed by:

Katja Breitkopf-Heinlein, Independent Researcher, Germany

Qing-Wu Yang,

Xinqiao Hospital, China

*Correspondence:

Manuela M. Santos

manuela.santos@umontreal.ca

${ }^{\dagger}$ These authors have contributed equally to this work.

Specialty section:

This article was submitted to

Gastrointestinal Sciences,

a section of the journal

Frontiers in Physiology

Received: 09 December 2017 Accepted: 16 February 2018

Published: 05 March 2018

Citation:

Layoun A, Samba-Mondonga M, Fragoso G, Calvé A and Santos MM

(2018) MyD88 Adaptor Protein Is

Required for Appropriate Hepcidin Induction in Response to Dietary Iron

Overload in Mice.

Front. Physiol. 9:159.

doi: 10.3389/fphys.2018.00159

\section{MyD88 Adaptor Protein Is Required for Appropriate Hepcidin Induction in Response to Dietary Iron Overload in Mice}

\author{
Antonio Layoun ${ }^{1,2 t}$, Macha Samba-Mondonga ${ }^{1,2 \dagger}$, Gabriela Fragoso ${ }^{1}$, Annie Calvé ${ }^{1}$ and \\ Manuela M. Santos ${ }^{1,2 *}$ \\ 'Nutrition and Microbiome Laboratory, Centre de Recherche du Centre Hospitalier de l'Université de Montréal, Montréal, \\ QC, Canada, ${ }^{2}$ Département de Médecine, Université de Montréal, Montréal, QC, Canada
}

Iron homeostasis is tightly regulated to provide virtually all cells in the body, particularly red blood cells, with this essential element while defending against its toxicity. The peptide hormone hepcidin is central to the control of the amount of iron absorbed from the diet and iron recycling from macrophages. Previously, we have shown that hepcidin induction in macrophages following Toll-like receptor (TLR) stimulation depends on the presence of myeloid differentiation primary response gene 88 (MyD88). In this study, we analyzed the regulation of iron metabolism in $\mathrm{MyD} 88^{-/-}$mice to further investigate MyD88 involvement in iron sensing and hepcidin induction. We show that mice lacking MyD88 accumulate significantly more iron in their livers than wild-type counterparts in response to dietary iron loading as they are unable to appropriately control hepcidin levels. The defect was associated with inappropriately low levels of Smad4 protein and Smad1/5/8 phosphorylation in liver samples found in the $M y D 88^{-/-}$mice compared to wild-type mice. In conclusion, our results reveal a previously unknown link between MyD88 and iron homeostasis, and provide new insights into the regulation of hepcidin through the iron-sensing pathway.

Keywords: iron, hepcidin, MyD88, Smad4, hemochromatosis, Bmp6, Bmp2

\section{INTRODUCTION}

Iron homeostasis in mammals is tightly regulated to meet body requirements while preventing iron toxicity, as iron can participate in the generation of harmful free radicals (Hentze et al., 2004; Sheftel et al., 2011). Most of this regulation takes place at the level of intestinal iron absorption, which is negatively controlled by levels of the peptide hormone hepcidin, encoded by the HAMP gene (Ganz and Nemeth, 2012). Hepcidin also plays a major role in regulating iron release from macrophages. These cells are responsible for iron recycling, with high hepcidin levels ultimately leading to iron accumulation in macrophages (Ganz and Nemeth, 2012). Erythropoietic activity, hypoxia, host defense, and multiple signals reflecting systemic iron stores and circulating levels converge to regulate hepcidin production, mostly in the liver, and affect body iron homeostasis (Huang et al., 2009). 
Two major pathways that contribute to hepcidin regulation are the inflammatory pathway mediated through IL-6/STAT3 signaling (Nemeth et al., 2004; Verga Falzacappa et al., 2007), and the iron-sensing pathway, which is mediated through BMP/SMAD signaling (Rishi et al., 2015). BMP/SMAD signaling is modulated in response to body iron status: increased dietary iron levels stimulate the production of bone morphogenetic protein 6 (BMP6) (Kautz et al., 2008; Meynard et al., 2009; Corradini et al., 2011), which then binds to heteromeric complexes containing type II and type I BMP receptors (BMPRI/II) (Parrow and Fleming, 2014). The binding of BMP6 to its receptors results in the recruitment and subsequent phosphorylation of SMADs 1,5 , and 8 (SMAD1/5/8). In turn, SMAD1/5/8 binds to SMAD4 to form a transcriptional complex that translocates to the nucleus and binds to specific DNA elements in the hepcidin promoter (Casanovas et al., 2009). While BMP6 is a key regulator of hepcidin expression and systemic iron homeostasis (Andriopoulos et al., 2009; Meynard et al., 2009), more recent work identified BMP2 as another BMP ligand indispensable for iron homeostasis in vivo that is non-redundant with BMP6 (Canali et al., 2017; Koch et al., 2017).

Hepcidin regulation through IL-6/STAT3 signaling occurs during inflammation or infection, when the host's innate immune response activates a sequence of events that limits iron availability by sequestering iron and down-regulating intestinal iron absorption (Weiss, 2009). Production of cytokines, particularly IL-6, leads to STAT3 phosphorylation, translocation into the nucleus, and binding to STAT3-recognizing DNA elements located in the hepcidin promoter (Verga Falzacappa et al., 2007).

Previously, we have shown that hepcidin induction in macrophages following stimulation of Toll-like receptors (TLRs) depends on the presence of myeloid differentiation primary response gene 88 (MyD88) (Layoun and Santos, 2012). In addition to macrophages, TLRs and MyD88 are also expressed in hepatocytes (Liu et al., 2002) and it has been demonstrated that LPS stimulation induces hepcidin expression in hepatocytes via a MyD88-dependent signaling pathway (Lee et al., 2017).

Almost all TLRs use MyD88 as a universal adapter protein to activate the transcription factor NF- $\mathrm{B}$ and cytokine production through the common MyD88-dependent signaling pathway. TRIF is another adapter protein used by TLR3 and TLR4 to activate NF- $\kappa \mathrm{B}$ through the TRIF-dependent signaling pathway (Yamamoto et al., 2003). Both MyD88 and Trif-deficient mice have impaired production of inflammatory cytokines (Takeuchi and Akira, 2010). In previous work, we showed that MyD88deficient mice are unable to sustain an acute hypoferremic response triggered by lipopolysaccharide (LPS), a TLR4 ligand (Layoun et al., 2012). The contribution of TLRs/MyD88 signaling for hepcidin expression through the inflammatory pathway has been further demonstrated using a variety of cellular and animal models (Wang et al., 2009; Xiong et al., 2016; Lee et al., 2017).

Since both the iron-sensing pathway and inflammatory pathways reveal overlap in hepcidin induction when converging at SMAD1/5/8 phosphorylation and SMAD4 binding, we investigated the potential role of $\mathrm{MyD} 88$ in iron sensing by analyzing iron metabolism in MyD88-deficient mice $\left(\mathrm{MyD} 88^{-/-}\right)$.

\section{MATERIALS AND METHODS}

\section{Animals}

This study was carried out in accordance with Canadian Council on Animal Care guidelines. The protocol was approved by the institutional Animal Care Committee of the CRCHUM.

C57BL/6 (B6) wild-type (Wt) and Trif-deficient mice

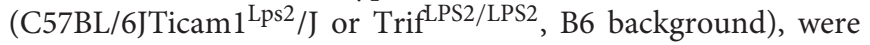
purchased from Jackson Laboratories (Bar Harbor, ME). $M y D 88^{-/-}$mice in the $\mathrm{B} 6$ genetic background were kindly provided by Dr. Shizuo Akira (Research Institute for Microbial Diseases, Osaka University, and Japan Science and Technology Agency, Tokyo, Japan) and were maintained as described previously (Adachi et al., 1998). Mice were maintained under standard 12:12 h light/dark conditions at the Centre de recherche du CHUM (CRCHUM). The animals used in the experiments were female and were permanently housed under specific pathogen-free conditions.

\section{Animal Treatments}

Mice were fed a commercial diet containing $\sim 200 \mathrm{mg}$ of iron per $\mathrm{kg}$ (Teklad Global 18\% protein rodent diet; Harlan Teklad, Madison, WI). Dietary iron overload was produced by feeding 8-week-old mice the same commercial diet supplemented with $25 \mathrm{~g}$ carbonyl iron per $\mathrm{kg}(2.5 \% \mathrm{wt} / \mathrm{wt}$ carbonyl iron; Sigma-Aldrich, St. Louis, MO, USA) for 2 weeks (Jiang et al., 2008). All animals were 10 -weeks old at the end of the experiments.

\section{Hematologic Measurements and Transferrin Saturation}

Red blood cell (RBC) count, hemoglobin (Hb), hematocrit (HCT), and mean corpuscular volume (MCV) were measured with an automated cell counter calibrated for murine samples

TABLE 1 | Primers used for qPCR analysis of mRNA levels.

\begin{tabular}{lll}
\hline Gene & & Sequence \\
\hline Actb & Forward & TGTTCCAACTGGGACGACA \\
& Reverse & GGTGTTGAGGTCTCAAA \\
Hamp & Forward & CCTATCTCCATCAACAGATG \\
& Reverse & AACAGATACCACACTGGGAA \\
Atoh8 & Forward & CACCATCAGCGCAGCCTTC \\
Id1 & Feverse & AATCCAGCAGGTCAGCAAAG \\
& Reverse & ACCCTGAACGGCGAGATCA \\
Bmp6 & Forward & TCGTCGGCTGGAACACATG \\
Bmp2 & Feverse & CCAGCAGGAGTCTCCCCACA \\
& Forward & GCCTGCACCCTGTTCTCTGA \\
& Reverse & ATGTCAAACACATATCCCTGGAA
\end{tabular}


( $\mathrm{ABC}$ vet counter; $\mathrm{ABX}$ Hématologie, Montpellier, France). Serum iron was assessed by colorimetric assay with the Kodak Ektachem DT60 system (Johnson \& Johnson, Ortho Clinical Diagnostics, Mississauga, ON).

\section{Measurement of Tissue Iron Concentration}

Spleen, and liver iron concentrations (LIC) were assessed by acid digestion of tissue samples, followed by iron quantification with atomic absorption spectroscopy (Wienk et al., 1997).

\section{Serum Ferritin Assay}

Ferritin was measured in serum with an enzyme-linked immunosorbent assay (ELISA) kit as per manufacturer's instructions (mouse Ferritin ELISA kit, Kamiya Biomedical, Seattle).

\section{Histology}

Liver tissue sections were stained with Perls' Prussian blue for ferric iron detection (iron stain kit; Sigma Immunochemicals).

\section{Quantitative RT-PCR}

Total RNA was isolated with Trizol reagent (Invitrogen, Burlington, $\mathrm{ON}$ ), and reverse transcription was performed with the Omniscript RT kit (QIAGEN, Mississauga, ON). mRNA expression levels were measured by real-time PCR in a Rotor Gene 3000 Real Time DNA Detection System (Montreal Biotech, Kirkland, QC) with QuantiTect SYBRGreen I PCR kits (QIAGEN, Mississauga, ON) as described (Makui et al., 2005). The primers used in this study are presented in Table 1. Expression levels were normalized to the housekeeping gene $\beta$-actin (Actb).

\section{SDS-PAGE and Western Blot Analysis}

Livers were removed, rinsed in ice-cold PBS, and used to prepare liver nuclear extracts with Nuclear Extract Kits (Active Motif, Carlsbad, CA). Total cell lysates and, when indicated, nuclear protein extracts were separated by $10 \%$ SDS-PAGE and blotted onto nitrocellulose membranes (BioRad Laboratories, Mississauga, Ontario). The membranes were immunoblotted with the following antibodies: ferritin (FTH1) (1:1000) (Alpha Diagnostic International, San Antonio, TX), phospho-Smad5 (1:1000) (Abcam, Cambridge, MA), Smad1
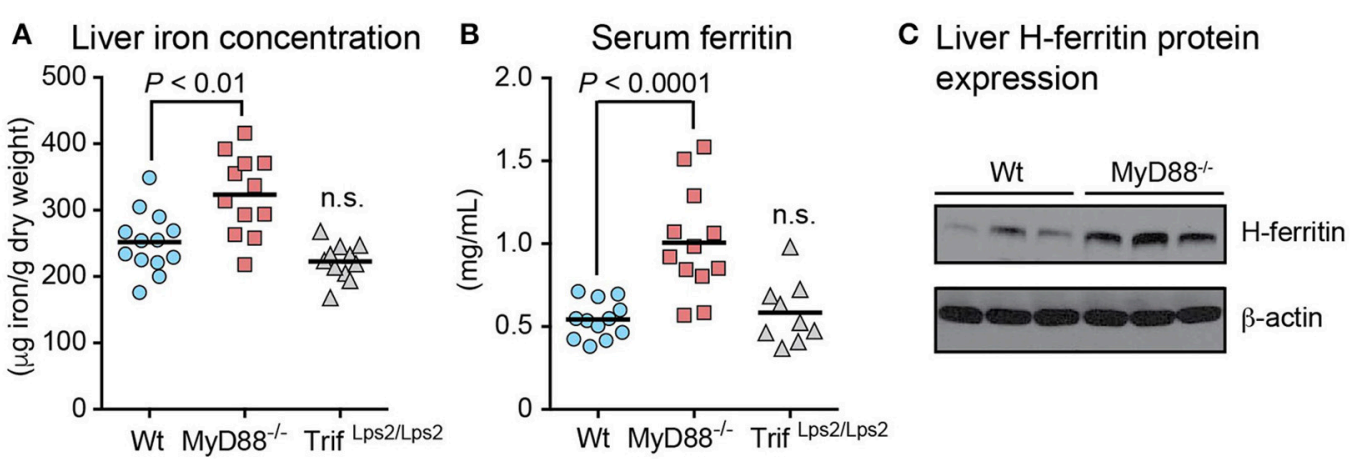

D

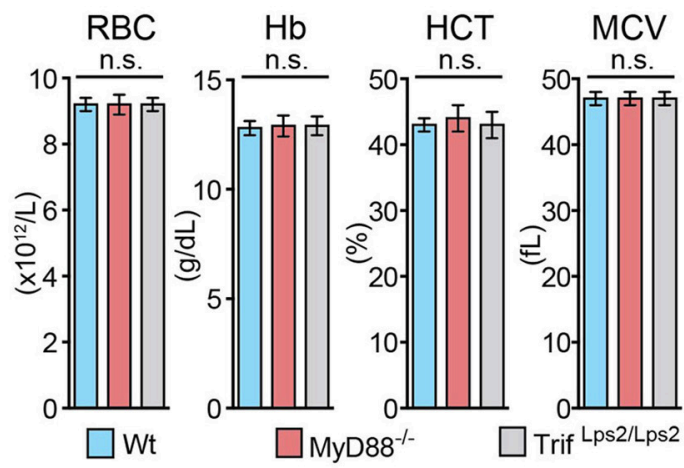

E

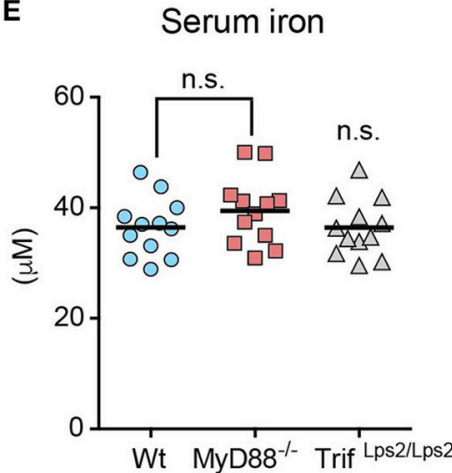

FIGURE 1 | Increased liver iron stores with normal erythropoietic parameters in MyD88-deficient mice. Wild-type (Wt), MyD88-deficient (MyD88 ${ }^{-/-}$) and Trif-deficient (Trif Lps2/Lps2) mice were fed a standard diet. (A) Liver iron concentration measured by atomic absorption spectroscopy. (B) Serum ferritin levels. (C) Representative western blot showing $\mathrm{H}$-ferritin levels in the liver of $\mathrm{Wt}$ and $\mathrm{MyD} 88^{-/-}$mice. The blot was stripped and reprobed with an antibody to $\beta$-actin as loading control. (D) Erythroid parameters: red blood cells (RBC), hemoglobin ( $\mathrm{Hb}$ ), hematocrit (HCT), and mean corpuscular volume (MCV). Results are presented as mean $\pm \mathrm{SEM}$. (E) Serum iron. Results are presented as combined data from two independent experiments performed with $n \geq 5$ mice per group. Statistical analysis was performed with one-way ANOVA. n.s., not significant compared to Wt mice. 
(1:1000) (Cell Signaling, Danvers, MA), Smad4 (1:1000) (Santa Cruz Biotechnology, Santa Cruz, CA), phospho-Stat3 (1:1000) (Cell Signaling, Danvers, MA), Stat3 (1:1000) (Cell Signaling, Danvers, MA), HDAC1 (1:1000) (Santa Cruz, Biotechnology, Santa Cruz, CA), $\beta$-Tubulin (1:1000) (Cell Signaling, Danvers, MA), and $\beta$-actin (1:10000) (Abcam, Cambridge, MA). Anti-rabbit IgG (1:5000) (Cell Signaling, Danvers, MA) or anti-mouse IgG (1:5000) (GE Healthcare, Amersham Biosciences, Baie d'Urfe, QC, Canada) was used as secondary antibody. Antigen-antibody complexes were visualized with the ECL Western Blotting Detection Reagent (GE Healthcare).

\section{Statistical Analysis}

All statistics were calculated with Prism software (GraphPad, San Diego, CA), with a pre-specified significant $P$-value of 0.05 . Multiple comparisons were evaluated statistically by oneway analysis of variance (ANOVA) followed by the Bonferroni multiple comparison test.

\section{RESULTS}

\section{Increased Liver Iron Stores in MyD88-/- Mice}

We have previously reported that mice deficient in MyD88, unlike Trif-deficient mice, are unable to maintain LPS-induced, acute hypoferremic response as they fail to divert iron from the circulation into the spleen (Layoun et al., 2012). To further investigate the role of MyD88 in iron metabolism, we first examined MyD88-deficient mice and assessed iron levels in the liver, the major iron storage organ in the body. Compared to Wt and Trif-deficient mice that were used as controls, $M y D 88^{-/-}$ animals presented consistently higher iron concentrations in the liver (Figure 1A). In addition, $M y D 88^{-/-}$mice had approximately twice the level of serum ferritin (Figure 1B) and higher levels of hepatic $\mathrm{H}$-ferritin protein (Figure 1C) than the levels observed in Wt mice, further confirming that MyD88 deficiency results in elevated liver iron storage. The increased iron concentration in $M y D 88^{-/-}$livers was not due to altered erythropoiesis or circulating iron since the erythroid parameters
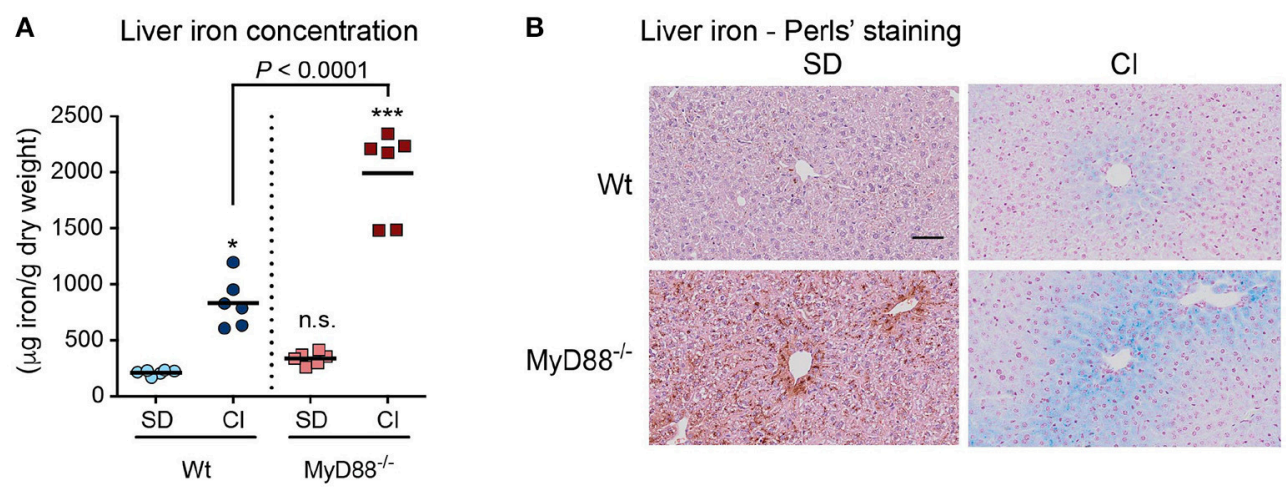

C Spleen iron concentration

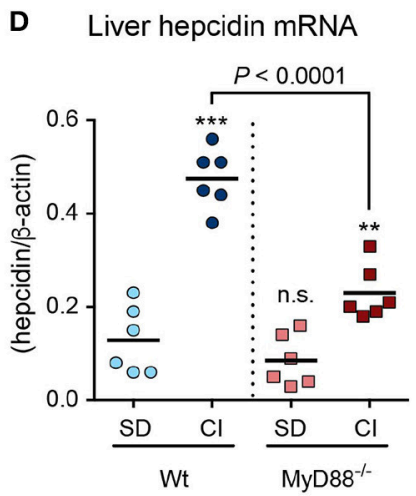

E Liver hepcidin/LIC ratio
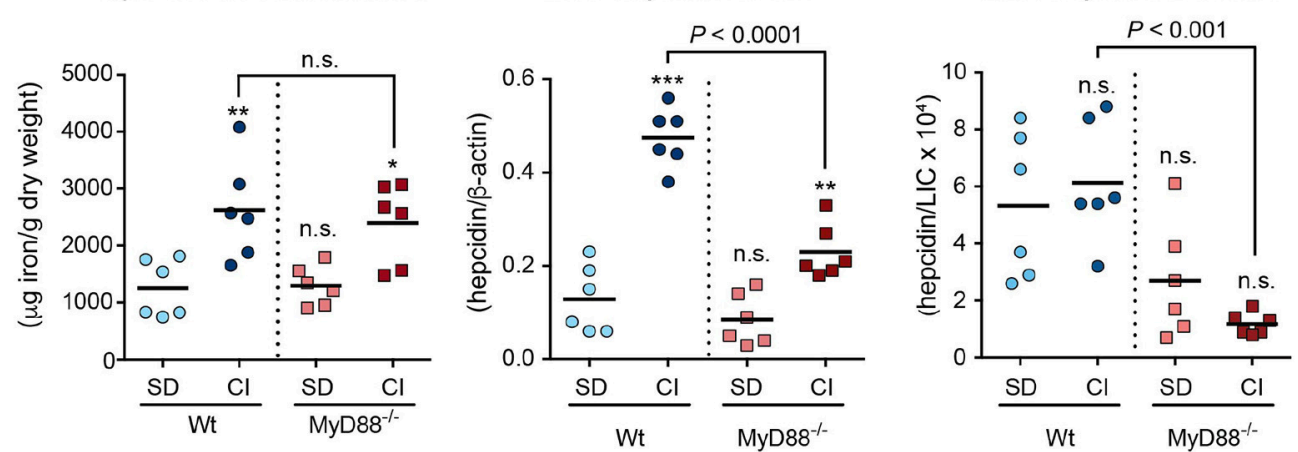

FIGURE 2 | Inability to appropriately upregulate hepcidin in response to iron challenge in MyD88-deficient mice. Wild-type (Wt) and MyD88-deficient (MyD88-/-) mice were fed a standard diet (SD) or carbonyl iron supplemented diet (CI) for 2 weeks. (A) Liver iron concentration measured by atomic absorption spectroscopy. (B) Ferric iron staining in liver sections. Left panels: Basal iron levels in the liver of mice fed SD, detected by DAB-enhanced Perl's staining (Smith et al., 1997) (brown) with hematoxylin (blue) and eosin counterstaining. Right panels: Iron levels in the liver of mice fed Cl, detected by Perls' Prussian blue staining. Scale bar $=50 \mu \mathrm{m}$. (C) Spleen iron concentration. (D) Liver hepcidin mRNA levels. (E) Ratio hepcidin/liver iron concentration (LIC). Results are representative of a minimum of three independent experiments using $n=4-6$ mice per group in each experiment. Statistical analysis was performed with one-way ANOVA. ${ }^{*} P<0.01,{ }^{*} P<0.001$, and ${ }^{\star \star \star} P<0.0001$, compared with mice fed SD; n.s., not significant. 


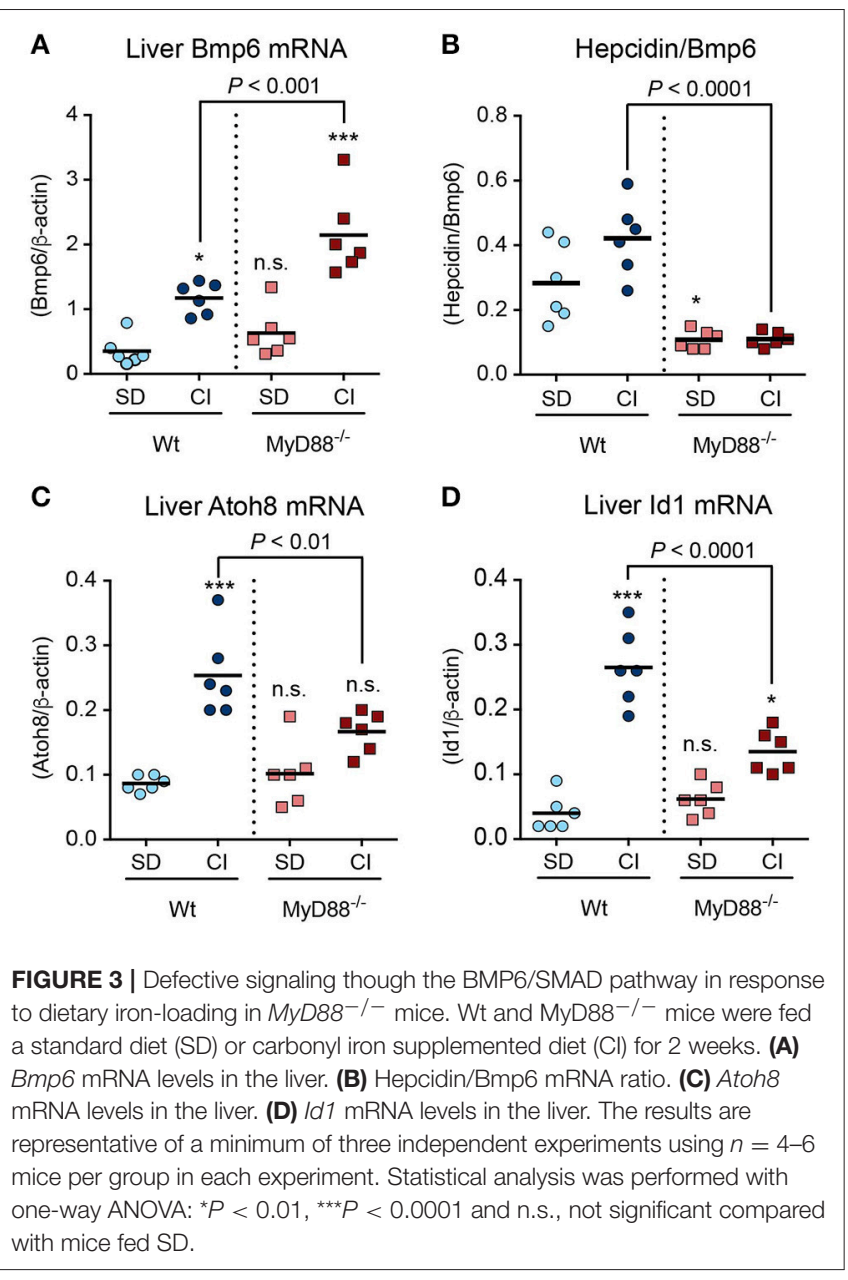

and serum iron levels in $M y D 88^{-/-}$mice were similar to those in Wt and Trif-deficient mice (Figures 1D,E).

\section{MyD88-/- Mice Exhibit Inability to Appropriately Regulate Hepcidin in Response to Dietary Iron Loading}

Next, we investigated whether MyD88 deficiency might impact the regulation of hepcidin with consequent storage of excess iron in the liver. Mice were challenged with an iron-enriched diet for 2 weeks to mimic chronic iron overload. This treatment resulted in a 5.6-fold increase of iron accumulation in the livers of $\mathrm{MyD} 88^{-/-}$mice compared to the 3.5-fold increase observed in Wt mice (Figure 2A), and was further confirmed by Perls' staining for ferric iron in liver samples (Figure 2B). In contrast, iron levels in the spleen of iron-loaded $M y D 88^{-/-}$ mice were similar to Wt mice (Figure 2C), indicating an inability to proportionately accumulate iron in this organ. These results suggest that $M y D 88^{-/-}$mice may have a defect in adjusting the levels of the iron regulatory hormone hepcidin (Ganz and Nemeth, 2012); therefore, we next assessed mRNA expression levels of liver hepcidin. Despite showing increased hepcidin mRNA expression upon chronic dietary iron-loading,
A

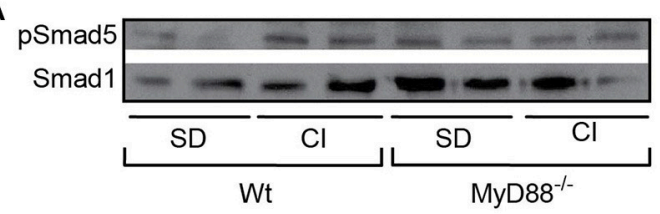

B

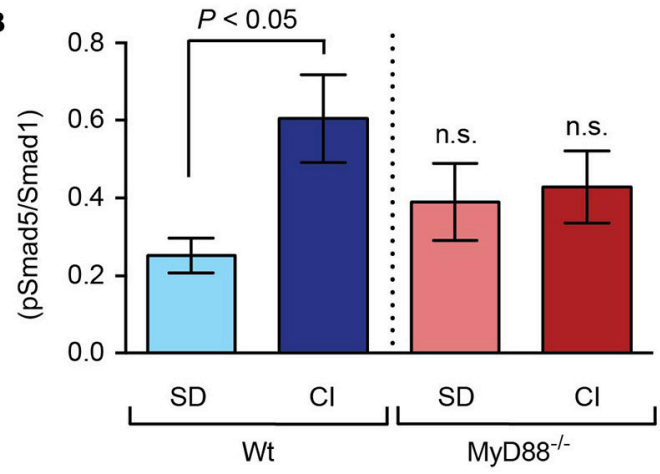

FIGURE 4 | Lack of Smad5 phosphorylation in response to dietary iron-loading in $\mathrm{MyD} 88^{-/}$mice. Liver nuclear extracts from mice fed standard $\operatorname{diet}(\mathrm{SD})$ and carbonyl iron supplemented diet (CI) were analyzed by western blots. (A) One representative blot is presented that was probed with antibodies against phosphorylated Smad5 (pSmad5) and total Smad1. (B) Graphic depicting densitometric quantification of western blots from three independent experiments. Statistical analysis was performed with one-way ANOVA: n.s., not significant compared with Wt mice fed the same diet.

$M y D 88^{-/-}$mice have comparatively lower levels of hepcidin than iron-loaded Wt mice (Figures 2D,E).

Altogether, our data suggest that mice lacking functional MyD88 adaptor molecules present insufficient hepcidin induction in response to dietary iron loading.

\section{Defective Signaling Through the BMP/SMAD Pathway in MyD88-/- Mice}

In vivo, iron loading induces the expression of Bmp6 mRNA in the liver (Kautz et al., 2008), which is believed to be an initial step in the activation of the BMP/SMAD1/5/8 signaling cascade for hepcidin induction in response to iron overloading (Meynard et al., 2009). Thus, we next sought to understand whether BMP6 activation was compromised in $M y D 88^{-/-}$mice. Liver Bmp6 mRNA expression was induced by iron in both Wt and $M y D 88^{-/-}$mice, and was even higher in iron-loaded $M y D 88^{-/-}$ mice, demonstrating that the absence of MyD88 did not affect the induction of Bmp6 (Figure 3A). Since BMP2 has also been shown to be necessary for iron homeostasis in vivo (Canali et al., 2017; Koch et al., 2017), we also measured Bmp2 mRNA expression in the liver. We found that Bmp2 mRNA levels were induced by dietary iron to similar levels in both Wt and $M y D 88^{-/-}$ mice (Supplementary Figure 1). However, induction of BMP ligands was not accompanied by the expected rise in hepcidin. Consequently, the ratio between Hamp mRNA and Bmp6 mRNA was persistently lower in $M y D 88^{-/-}$mice compared to Wt mice (Figure 3B), and indicated a defect in the downstream signaling transmission initiated by Bmp6. Measurements of the mRNA 
levels of Atoh8 and Id1, two genes shown to be induced by iron through the BMP6/SMAD4 pathway (Kautz et al., 2008), further reinforced the idea that the main defect in $M y D 88^{-/-}$mice resides in the BMP6/SMAD4 down-stream signaling pathway of targeted genes. In fact, the mRNA levels of both genes were significantly lower in $M y D 88^{-/-}$mice compared to wild-type mice in response to dietary iron loading (Figures 3C,D).

BMP6 signals through the phosphorylation of SMAD proteins 1, 5, and 8 (Kersten et al., 2005), which translocate into the nucleus where they regulate the transcription of specific target genes, including hepcidin (Pantopoulos et al., 2012). Hence, we next analyzed phosphorylation levels of Smad5 in liver nuclear extracts from dietary iron-loaded $M y D 88^{-/-}$and $\mathrm{Wt}$ mice. Dietary iron loading induced phosphorylation of liver Smad5 proteins in Wt mice, as expected (Besson-Fournier et al., 2017; Canali et al., 2017). In contrast, despite higher hepatic iron levels found in $M y D 88^{-/-}$mice, no significant differences regarding Smad5 phosphorylation were detected in this mouse strain when comparing the standard diet with the carbonyl iron supplemented diet (Figures 4A,B).

For the activation signal to be successfully transmitted, phosphorylated SMAD1/5/8 form heteromeric complexes with the common mediator SMAD4 before translocation into the nucleus. Thus, we next investigated whether the lack of MyD88 affected the amount of Smad4. As shown in Figures 5A,B, we found that Smad4 protein levels in nuclear extracts were significantly lower in $M y D 88^{-/-}$mice compared to Wt mice, both in mice fed the standard diet and the carbonyl iron supplemented diet (Supplementary Figure 2).

To exclude the influence of MyD88 mediated inflammatory response on hepcidin expression we measured Stat3 phosphorylation levels in liver nuclear extracts. As shown in Figures 6A,B, no significant differences were found regarding Stat3 phosporylation levels between mouse strains (Wt vs. $\mathrm{MyD} 88^{-/-}$mice) or treatments (standard diet vs. carbonyl iron supplemented diet).

\section{DISCUSSION}

Previous studies have shown that MyD88 is required for a sustained LPS-induced hypoferremic response (Layoun et al., 2012). To further study the role of MyD88 in iron sensing in vivo, we analyzed iron metabolism in MyD88-deficient mice. Unlike Trif-deficient mice, $M y D 88^{-/-}$mice kept on a standard diet exhibited increased iron levels in the liver compared to $\mathrm{Wt}$ mice. Accordingly, $M y D 88^{-/-}$mice also showed higher serum and liver ferritin levels. Ferritin can be abnormally elevated in a wide range of disease states including malignancy, diabetes, infection, inflammation, and chronic iron-overload syndromes (Torti and Torti, 1994; Lee and Means, 1995; Turnbull et al., 1997; Acton et al., 2006; Jacobs et al., 2009). Since $M y D 88^{-/-}$ mice present higher liver iron stores without activation of Stat3 phosphorylation, the increased ferritin levels in the serum most likely reflected excess iron accumulation in the liver.

High levels of iron and ferritin reflecting increased iron stores are encountered in the liver and serum of a number of disorders
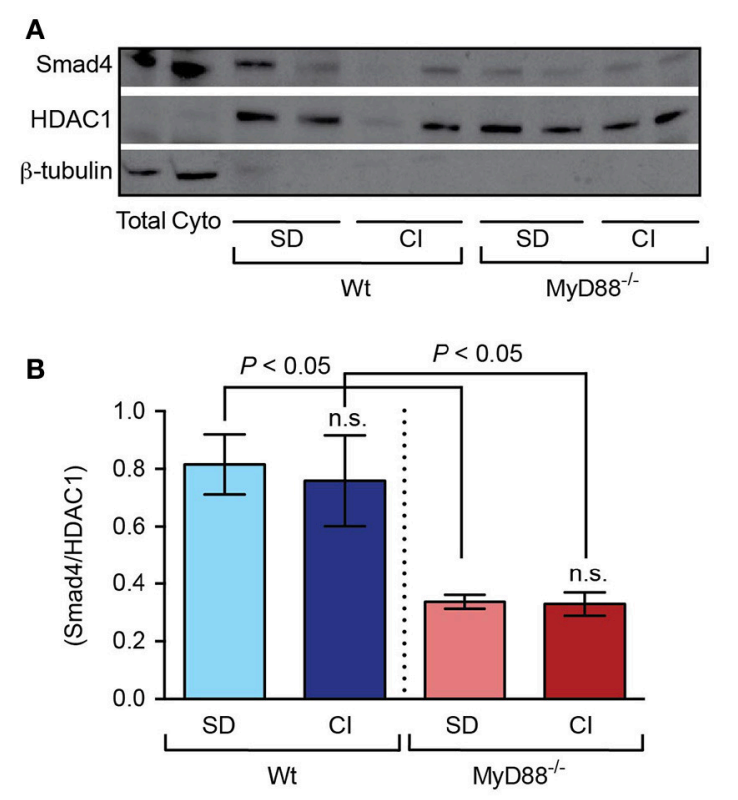

FIGURE 5 | Defective signaling in response to dietary iron-loading in MyD88-/- mice involves diminished Smad4 protein in the liver. Liver nuclear extracts from mice fed standard diet (SD) and carbonyl iron supplemented diet (Cl) were analyzed by western blots. (A) One representative blot probed with an antibody against Smad4. Blots were stripped and reprobed with an antibody to HDAC1 (nuclear marker) and to $\beta$-tubulin (cytoplasmic marker). Total, liver total extract; Cyt, liver cytoplasmic extract. (B) Graphic depicting densitometric quantification of western blots from three independent experiments. Statistical analysis was performed with one-way ANOVA: n.s., not significant compared with mice fed SD.

that are collectively called iron-loading anemias. These include thalassemia syndromes, congenital dyserythropoietic anemias, and sideroblastic anemias, all of which have the presence of robust but inefficient erythropoiesis (Nemeth and Ganz, 2006). To rule out the possibility that $M y D 88^{-/-}$iron burden was due to augmented erythropoiesis activity (Papanikolaou and Pantopoulos, 2017) we measured several erythroid parameters and found no differences between $M y D 88^{-/-}$, Wt and Trifdeficient mice, hence ruling out a possible defect in erythropoiesis activity in $M y D 88^{-/-}$mice.

Increased body iron stores are also found in the different forms of hereditary hemochromatosis (HH) (Pietrangelo, 2006). All these hereditary disorders have a common feature of inappropriately low hepcidin levels with consequential excessive iron accumulation in the liver with relative sparing of the spleen. These features are reproduced in other mouse models with hepcidin deficiency, such as $\mathrm{Hfe}^{-/-}$and $\mathrm{Hjv}^{-/-}$mice (Makui et al., 2005; Kent et al., 2015). Similarly, MyD88-/mice challenged with dietary iron were unable to appropriately control hepcidin levels, resulting in significantly higher liver iron accumulation when compared to Wt mice. These results suggest a defect in $M y D 88^{-/-}$mice in the iron-sensing pathway leading to insufficient hepcidin induction. However, unlike other mouse models of iron overload, iron accumulation in the liver occurs in the absence of increased serum iron levels. A possible explanation 
A

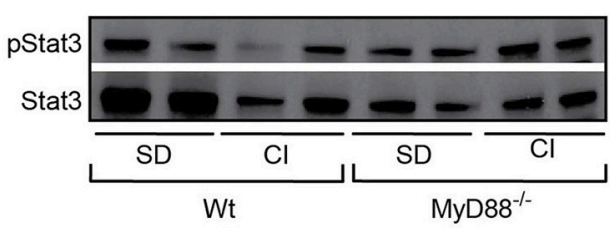

B

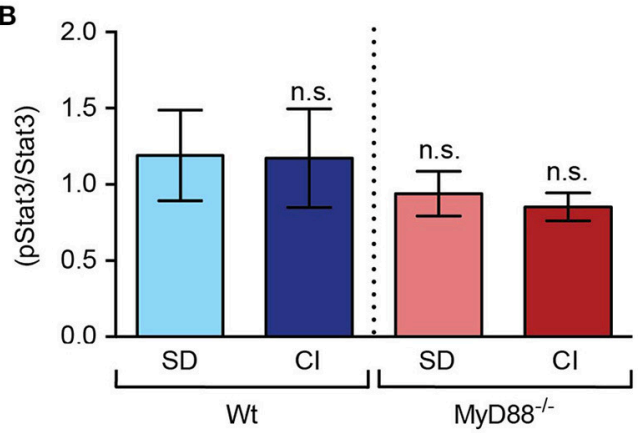

FIGURE 6 | Stat3 phosphorylation in response to dietary iron-loading remains unchanged in both Wt and in MyD88-/- mice. Liver nuclear extracts from mice fed standard diet (SD) and carbonyl iron supplemented diet (Cl) were analyzed by western blots. (A) One representative blot probed with antibodies against phosphorylated Stat3 (pStat3) and total Stat3 (Stat3). (B) Graphic depicting densitometric quantification of western blots from three independent experiments. Statistical analysis was performed with one-way ANOVA: n.s., not significant compared with mice fed SD.

for this difference could be enhanced ability to transport iron into hepatocytes involving heightened expression and/or activity of iron uptake molecules.

In vivo, hepcidin expression correlates with Bmp6 gene expression in mice fed an iron-enriched or iron-deficient diet (Andriopoulos et al., 2009; Ryan et al., 2010). Accordingly, Bmp6deficient mice present insufficient hepcidin levels and consequent massive iron overload (Meynard et al., 2009). Besides Bmp6, there is increasing evidence that Bmp2 also plays an important role in iron homeostasis, since mice models lacking expression of Bmp2 in liver endothelial cells develop an iron loading phenotype similar to Bmp6 ${ }^{-/-}$mice (Canali et al., 2017; Koch et al., 2017). In $M y D 88^{-/-}$mice, we show that the appropriate Bmp6 and $\mathrm{Bmp} 2$ induction in response to iron loading is disassociated from hepcidin production. The defect was associated with the levels of Smad4 protein found in nuclear extracts from liver samples, which were diminished by about half in the $M y D 88^{-/-}$mice compared to Wt mice. This finding is in line with previously reported data that described Smad4 deficiency in mice causing iron overload due to insufficient hepcidin activation (Wang et al., 2005). In addition, our results indicate that the levels of Smad5 phosphorylation were too low in $M y D 88^{-/-}$mice when taking into account iron and Bmp6 levels in the liver (Kautz et al., 2008), suggesting a potential defect in Smad1/5/8 phosphorylation.

A connection between the SMAD and the MyD88 pathway has been reported that concerns the role of SMAD6 as a negative regulator of the transforming growth factor $\beta$ (TGF- $\beta$ ) family signaling pathway, which also involves BMP (Goto et al., 2007; Lee et al., 2011). The study by Lee et al. showed SMAD6 physically interacts with MyD88. Potentially, MyD88 interactions with SMAD4 and SMAD6 may be relevant for the regulation of hepcidin, given the reported role of SMAD6 in hepcidin suppression (Lee et al., 2017).

More recently, interaction between the TLR4/MyD88 and BMP2-induced BMP/SMAD4 signaling has been demonstrated in osteoblasts, where the two pathways were found to play conflicting roles in the regulation of BMP-2-induced osteoblast differentiation (Huang et al., 2013). In their study, Huang et al. showed that LPS-mediated inflammatory environment inhibits BMP-2-induced osteogenic differentiation through the crosstalk between TLR4/MyD88/NF- $\kappa \mathrm{B}$ and BMP/SMAD signaling, which contrasts with our present findings. However, cross-talk between the inflammatory and SMAD pathways could be cell-context specific, since receptor expression seems to modulate the specificity of TGF- $\beta$ signaling pathways (Murakami et al., 2009). In fact, stimulation of SMAD1/5/8 signaling and hepcidin induction during inflammation by other TGF- $\beta$ superfamily members, such as activin B (Besson-Fournier et al., 2012), has been shown to be unique in liver cells (Canali et al., 2016). The exact nature of the cross-talk between MyD88 and SMAD pathways remains to be defined, and further studies are needed to understand whether MyD88 may, for example, stabilize SMAD4 and/or have a role in the translocation of the SMAD4/phosphorylated SMAD1/5/8 complexes into the nucleus.

In summary, we report here for the first time that MyD88 is critical for appropriate nuclear Smad4 protein expression, Smad1/5/8 phosphorylation, and for the induction of hepcidin upon challenge with dietary iron. Together, our data identify a new role for MyD88 as a potential key molecule in the BMP signaling pathway mediated by SMAD proteins.

\section{AUTHOR CONTRIBUTIONS}

MS-M, AL, GF, and AC contributed to the investigation, validation, methodology, and formal analysis. MS-M, AL, and MS additionally contributed to the conceptualization and writing of the original draft of the manuscript. MS additionally contributed to the visualization, supervision, and funding acquisition of the study.

\section{ACKNOWLEDGMENTS}

This work was supported by grants from the Canadian Institutes of Health Research (CIHR, grant no MOP123246) and Natural Sciences and Engineering Research Council of Canada (NSERC, grant no 298515-2011) to MS. AL and MS-M are recipients of PhD scholarships from the NSERC and the Institut du Cancer de Montreal, respectively. We wish to thank Jacqueline Chung for editing the manuscript.

\section{SUPPLEMENTARY MATERIAL}

The Supplementary Material for this article can be found online at: https://www.frontiersin.org/articles/10.3389/fphys. 2018.00159/full\#supplementary-material 


\section{REFERENCES}

Acton, R. T., Barton, J. C., Passmore, L. V., Adams, P. C., Speechley, M. R., Dawkins, F. W., et al. (2006). Relationships of serum ferritin, transferrin saturation, and HFE mutations and self-reported diabetes in the Hemochromatosis and Iron Overload Screening (HEIRS) study. Diabetes Care 29, 2084-2089. doi: $10.2337 / \mathrm{dc} 05-1592$

Adachi, O., Kawai, T., Takeda, K., Matsumoto, M., Tsutsui, H., Sakagami, M., et al. (1998). Targeted disruption of the MyD88 gene results in loss of IL-1- and IL-18-mediated function. Immunity 9, 143-150. doi: 10.1016/S1074-7613(00)80596-8

Andriopoulos, B. Jr., Corradini, E., Xia, Y., Faasse, S. A., Chen, S., Grgurevic, L., et al. (2009). BMP6 is a key endogenous regulator of hepcidin expression and iron metabolism. Nat. Genet. 41, 482-487. doi: 10.1038/ng.335

Besson-Fournier, C., Gineste, A., Latour, C., Gourbeyre, O., Meynard, D., Martin, P., et al. (2017). Hepcidin upregulation by inflammation is independent of Smad1/5/8 signaling by activin B. Blood 129, 533-536. doi: 10.1182/blood-2016-10-748541

Besson-Fournier, C., Latour, C., Kautz, L., Bertrand, J., Ganz, T., Roth, M. P., et al. (2012). Induction of activin B by inflammatory stimuli up-regulates expression of the iron-regulatory peptide hepcidin through Smad1/5/8 signaling. Blood 120, 431-439. doi: 10.1182/blood-2012-02-411470

Canali, S., Core, A. B., Zumbrennen-Bullough, K. B., Merkulova, M., Wang, C. Y., Schneyer, A. L., et al. (2016). Activin. B induces noncanonical SMAD1/5/8 signaling via BMP type I receptors in hepatocytes: evidence for a role in hepcidin induction by inflammation in male mice. Endocrinology 157, 1146-1162. doi: 10.1210/en.2015-1747

Canali, S., Wang, C. Y., Zumbrennen-Bullough, K. B., Bayer, A., and Babitt, J. L. (2017). Bone morphogenetic protein 2 controls iron homeostasis in mice independent of Bmp6. Am. J. Hematol. 92, 1204-1213. doi: 10.1002/ajh.24888

Casanovas, G., Mleczko-Sanecka, K., Altamura, S., Hentze, M. W., and Muckenthaler, M. U. (2009). Bone morphogenetic protein (BMP)-responsive elements located in the proximal and distal hepcidin promoter are critical for its response to HJV/BMP/SMAD. J. Mol. Med. 87, 471-480. doi: 10.1007/s00109-009-0447-2

Corradini, E., Meynard, D., Wu, Q., Chen, S., Ventura, P., Pietrangelo, A., et al. (2011). Serum and liver iron differently regulate the bone morphogenetic protein 6 (BMP6)-SMAD signaling pathway in mice. Hepatology 54, 273-284. doi: $10.1002 /$ hep. 24359

Ganz, T., and Nemeth, E. (2012). Hepcidin and iron homeostasis. Biochim. Biophys. Acta 1823, 1434-1443. doi: 10.1016/j.bbamcr.2012.01.014

Goto, K., Kamiya, Y., Imamura, T., Miyazono, K., and Miyazawa, K. (2007). Selective inhibitory effects of Smad6 on bone morphogenetic protein type I receptors. J. Biol. Chem. 282, 20603-20611. doi: 10.1074/jbc.M702100200

Hentze, M. W., Muckenthaler, M. U., and Andrews, N. C. (2004). Balancing acts: molecular control of mammalian iron metabolism. Cell 117, 285-297. doi: 10.1016/S0092-8674(04)00343-5

Huang, H., Constante, M., Layoun, A., and Santos, M. M. (2009). Contribution of STAT3 and SMAD4 pathways to the regulation of hepcidin by opposing stimuli. Blood 113, 3593-3599. doi: 10.1182/blood-2008-08-173641

Huang, R. L., Yuan, Y., Zou, G. M., Liu, G., Tu, J., and Li, Q. (2013). LPSstimulated inflammatory environment inhibits BMP-2-induced osteoblastic differentiation through crosstalk between TLR4/MyD88/NF-kB and BMP/Smad signaling. Stem Cells Dev. 23, 277-289. doi: 10.1089/scd.2013.0345

Jacobs, E. M., Hendriks, J. C., Van Deursen, C. T., Kreeftenberg, H. G., De Vries, R. A., Marx, J. J., et al. (2009). Severity of iron overload of proband determines serum ferritin levels in families with HFE-related hemochromatosis: the HEmochromatosis FAmily Study. J. Hepatol. 50, 174-183. doi: 10.1016/j.jhep.2008.08.014

Jiang, W., Constante, M., and Santos, M. M. (2008). Anemia upregulates lipocalin 2 in the liver and serum. Blood Cells Mol. Dis. 41, 169-174. doi: 10.1016/j.bcmd.2008.04.006

Kautz, L., Meynard, D., Monnier, A., Darnaud, V., Bouvet, R., Wang, R. H., et al. (2008). Iron regulates phosphorylation of Smad1/5/8 and gene expression of Bmp6, Smad7, Id1, and Atoh8 in the mouse liver. Blood 112, 1503-1509. doi: 10.1182/blood-2008-03-143354

Kent, P., Wilkinson, N., Constante, M., Fillebeen, C., Gkouvatsos, K., Wagner, J., et al. (2015). Hfe and Hjv exhibit overlapping functions for iron signaling to hepcidin. J. Mol. Med. 93, 489-498. doi: 10.1007/s00109-0151253-7

Kersten, C., Sivertsen, E. A., Hystad, M. E., Forfang, L., Smeland, E. B., and Myklebust, J. H. (2005). BMP-6 inhibits growth of mature human B cells; induction of Smad phosphorylation and upregulation of Id1. BMC Immunol. 6:9. doi: 10.1186/1471-2172-6-9

Koch, P. S., Olsavszky, V., Ulbrich, F., Sticht, C., Demory, A., Leibing, T., et al. (2017). Angiocrine Bmp2 signaling in murine liver controls normal iron homeostasis. Blood 129, 415-419. doi: 10.1182/blood-2016-07729822

Layoun, A., Huang, H., Calvé, A., and Santos, M. M. (2012). Toll-like receptor signal adaptor protein MyD88 is required for sustained endotoxin-induced acute hypoferremic response in mice. Am. J. Pathol. 180, 2340-2350. doi: $10.1016 /$ j.ajpath.2012.01.046

Layoun, A., and Santos, M. M. (2012). Bacterial cell wall constituents induce hepcidin expression in macrophages through MyD88 signaling. Inflammation 35, 1500-1506. doi: 10.1007/s10753-012-9463-4

Lee, M. H., and Means, R.T. Jr. (1995). Extremely elevated serum ferritin levels in a university hospital: associated diseases and clinical significance. Am. J. Med. 98, 566-571. doi: 10.1016/S0002-9343(99)80015-1

Lee, Y. S., Kim, Y. H., Jung, Y. S., Kim, K. S., Kim, D. K., Na, S. Y., et al. (2017). Hepatocyte toll-like receptor 4 mediates lipopolysaccharide-induced hepcidin expression. Exp. Mol. Med. 49:e408. doi: 10.1038/emm.2017.207

Lee, Y. S., Park, J. S., Kim, J. H., Jung, S. M., Lee, J. Y., Kim, S. J., et al. (2011). Smad6-specific recruitment of Smurf E3 ligases mediates TGF- $\beta 1$ induced degradation of MyD88 in TLR4 signalling. Nat. Commun. 2:460. doi: $10.1038 /$ ncomms 1469

Liu, S., Gallo, D. J., Green, A. M., Williams, D. L., Gong, X., Shapiro, R. A., et al. (2002). Role of toll-like receptors in changes in gene expression and NF-KB activation in mouse hepatocytes stimulated with Lipopolysaccharide. Infect. Immun. 70, 3433-3442. doi: 10.1128/IAI.70.7.34333442.2002

Makui, H., Soares, R. J., Jiang, W., Constante, M., and Santos, M. M. (2005). Contribution of Hfe expression in macrophages to the regulation of hepatic hepcidin levels and iron loading. Blood 106, 2189-2195. doi: 10.1182/blood-2005-02-0629

Meynard, D., Kautz, L., Darnaud, V., Canonne-Hergaux, F., Coppin, H., and Roth, M. P. (2009). Lack of the bone morphogenetic protein BMP6 induces massive iron overload. Nat. Genet. 41, 478-481. doi: 10.1038/ng.320

Murakami, M., Kawachi, H., Ogawa, K., Nishino, Y., and Funaba, M. (2009). Receptor expression modulates the specificity of transforming growth factor- $\beta$ signaling pathways. Genes Cells 14, 469-482. doi: $10.1111 / j .1365-2443.2009 .01283 . x$

Nemeth, E., and Ganz, T. (2006). Hepcidin and iron-loading anemias. Haematologica 91, 727-732.

Nemeth, E., Rivera, S., Gabayan, V., Keller, C., Taudorf, S., Pedersen, B. K., et al. (2004). IL-6 mediates hypoferremia of inflammation by inducing the synthesis of the iron regulatory hormone hepcidin. J. Clin. Invest. 113, 1271-1276. doi: 10.1172/JCI200420945

Pantopoulos, K., Porwal, S. K., Tartakoff, A., and Devireddy, L. (2012). Mechanisms of mammalian iron homeostasis. Biochemistry 51, 5705-5724. doi: 10.1021/bi300752r

Papanikolaou, G., and Pantopoulos, K. (2017). Systemic iron homeostasis and erythropoiesis. IUBMB Life 69, 399-413. doi: 10.1002/iub.1629

Parrow, N. L., and Fleming, R. E. (2014). Bone morphogenetic proteins as regulators of iron metabolism. Annu. Rev. Nutr. 34, 77-94. doi: 10.1146/annurev-nutr-071813-105646

Pietrangelo, A. (2006). Molecular insights into the pathogenesis of hereditary haemochromatosis. Gut 55, 564-568. doi: 10.1136/gut.2005.078063

Rishi, G., Wallace, D. F., and Subramaniam, V. N. (2015). Hepcidin: regulation of the master iron regulator. Biosci. Rep. 35:e00192. doi: 10.1042/BSR20150014

Ryan, J. D., Ryan, E., Fabre, A., Lawless, M. W., and Crowe, J. (2010). Defective bone morphogenic protein signaling underlies hepcidin deficiency in HFE hereditary hemochromatosis. Hepatology 52, 1266-1273. doi: $10.1002 /$ hep. 23814

Sheftel, A. D., Mason, A. B., and Ponka, P. (2011). The long history of iron in the Universe and in health and disease. Biochim. Biophys. Acta 1820, 161-187. doi: 10.1016/j.bbagen.2011.08.002 
Smith, M. A., Harris, P. L., Sayre, L. M., and Perry, G. (1997). Iron accumulation in Alzheimer disease is a source of redox-generated free radicals. Proc. Natl. Acad. Sci. U.S.A. 94, 9866-9868. doi: 10.1073/pnas.94.18.9866

Takeuchi, O., and Akira, S. (2010). Pattern recognition receptors and inflammation. Cell 140, 805-820. doi: 10.1016/j.cell.2010.01.022

Torti, S. V., and Torti, F. M. (1994). Iron and ferritin in inflammation and cancer. Adv. Inorg. Biochem. 10, 119-137.

Turnbull, A. J., Mitchison, H. C., Peaston, R. T., Lai, L. C., Bennett, M. K., Taylor, R., et al. (1997). The prevalence of hereditary haemochromatosis in a diabetic population. QJM 90, 271-275. doi: 10.1093/qjmed/90.4.271

Verga Falzacappa, M. V., Vujic Spasic, M., Kessler, R., Stolte, J., Hentze, M. W., and Muckenthaler, M. U. (2007). STAT3 mediates hepatic hepcidin expression and its inflammatory stimulation. Blood 109, 353-358. doi: 10.1182/blood-2006-07-033969

Wang, L., Harrington, L., Trebicka, E., Shi, H. N., Kagan, J. C., Hong, C. C., et al. (2009). Selective modulation of TLR4-activated inflammatory responses by altered iron homeostasis in mice. J. Clin. Invest. 119, 3322-3328. doi: 10.1172/JCI39939

Wang, R. H., Li, C., Xu, X., Zheng, Y., Xiao, C., Zerfas, P., et al. (2005). A role of SMAD4 in iron metabolism through the positive regulation of hepcidin expression. Cell Metab. 2, 399-409. doi: 10.1016/j.cmet. 2005.10.010

Weiss, G. (2009). Iron metabolism in the anemia of chronic disease. Biochim. Biophys. Acta 1790, 682-693. doi: 10.1016/j.bbagen.2008.08.006
Wienk, K. J. H., Marx, J. J. M., Santos, M., Lemmens, A. G., Brink, E. J., Van Der Meer, R., et al. (1997). Dietary ascorbic acid raises iron absorption in anaemic rats through enhancing mucosal iron uptake independent of iron solubility in the digesta. Br. J. Nutrit. 77, 123-131. doi: 10.1079/BJN19970014

Xiong, X. Y., Liu, L., Wang, F. X., Yang, Y. R., Hao, J. W., Wang, P. F., et al. (2016). Toll-like receptor 4/MyD88-mediated signaling of hepcidin expression causing brain iron accumulation, oxidative injury, and cognitive impairment after intracerebral hemorrhage. Circulation 134, 1025-1038. doi: 10.1161/CIRCULATIONAHA.116.021881

Yamamoto, M., Sato, S., Hemmi, H., Hoshino, K., Kaisho, T., Sanjo, H., et al. (2003). Role of adaptor TRIF in the MyD88-independent toll-like receptor signaling pathway. Science 301, 640-643. doi: 10.1126/science.1087262

Conflict of Interest Statement: The authors declare that the research was conducted in the absence of any commercial or financial relationships that could be construed as a potential conflict of interest.

Copyright (๑) 2018 Layoun, Samba-Mondonga, Fragoso, Calvé and Santos. This is an open-access article distributed under the terms of the Creative Commons Attribution License (CC BY). The use, distribution or reproduction in other forums is permitted, provided the original author(s) and the copyright owner are credited and that the original publication in this journal is cited, in accordance with accepted academic practice. No use, distribution or reproduction is permitted which does not comply with these terms. 